\title{
Symmetries for scalarless scalar theories
}

\author{
Gianmassimo Tasinato \\ Department of Physics, Swansea University, Swansea SA2 8PP, United Kingdom
}

(Received 18 August 2020; accepted 10 September 2020; published 5 October 2020)

\begin{abstract}
We consider theories containing scalar fields interacting with vector or with tensor degrees of freedom, equipped with symmetries that prevent the propagation of linearized scalar excitations around solutions of the equations of motion. We first study the implications of such symmetries for building vector theories that break Abelian gauge invariance without necessarily exciting longitudinal scalar fluctuations in flat space. We then examine scalar-tensor theories in curved space, and relate the symmetries we consider with a nonlinear realization of broken spacetime symmetries acting on scalar modes. We determine sufficient conditions on the spacetime geometry to avoid the propagation of scalar fluctuations. We analyze linearized perturbations around spherically symmetric black holes, proving the absence of scalar excitations, and pointing out modifications in the dynamics of spin-2 fluctuations with respect to Einstein gravity. We then study consequences of this setup for the dark energy problem, determining scalar constraints on cosmological configurations that can lead to self-accelerating universes whose expansion is insensitive to the value of the bare cosmological constant.
\end{abstract}

DOI: 10.1103/PhysRevD.102.084009

\section{INTRODUCTION}

In this work we examine covariant theories describing scalar fields interacting with themselves, with vectors, or with tensors, where symmetries prevent the propagation of linearized scalar excitations around solutions of the equations of motion. Our motivations for studying these systems are the following:

They can constitute examples of theories of gravity alternative to general relativity (GR) that automatically avoid fifth force constraints, since they only admit a massless spin- 2 propagating mode. In fact, if scalar fluctuations do not propagate, long range scalar interactions are absent. These systems circumvent the Lovelock theorem [1] by spontaneously breaking Lorentz invariance by a nontrivial profile for the scalar background configuration.

These theories might avoid instabilities associated with scalar fluctuations in scalar-tensor systems, as for example graviton decay into dark energy [2], or scalar instabilities around spherically symmetric black holes [3-7]. Yet, the dynamics of tensor fluctuations can be distinct with respect to GR, making them distinguishable from Einstein gravity.

Published by the American Physical Society under the terms of the Creative Commons Attribution 4.0 International license. Further distribution of this work must maintain attribution to the author(s) and the published article's title, journal citation, and DOI. Funded by SCOAP ${ }^{3}$.
Symmetries can limit the number of allowed interactions for the setup under examination, and protect their structure under classical and quantum corrections. The structure of the corresponding theories can lead to new perspectives for the dark energy problem, and for explaining the smallness of the present-day cosmological acceleration rate [8].

Examples of scalar-tensor or massive gravity theories where scalar fluctuations do not propagate have already been discussed in the literature (see, e.g., [9-13]), usually performing a Hamiltonian analysis aimed at determining the constraints that avoid the propagation of scalar modes. In many of the existing examples, scalar fluctuations do not propagate in a unitary gauge, where an homogeneous, timedependent-only configuration for the background scalar field is selected. In this work, we address the problem of determining scalarless scalar-tensor theories of gravity making use of symmetry principles that guide us for building covariant scalarless theories and for better understanding the dynamics of the propagating modes. We focus on symmetries first explored in $[14,15]$ that arise when taking certain limits of Dirac-Born-Infeld (DBI)-Galileon interactions [16], and we further develop and expand previous results. We aim to better understand how such scalarless interactions arise when breaking gauge or spacetime symmetries in vector or tensor theories, with the scalar playing the role of Goldstone bosons of such symmetries. We then study the consequences of our results for spherically symmetric configurations and for cosmology. We proceed as follows:

(i) We start in Sec. II considering scalar theories in flat space in the absence of gravity and studying a 
field-dependent coordinate reparametrization $[\pi(x)$ is the scalar field, and $\omega^{\mu}$ an arbitrary infinitesimal constant vector]

$$
x^{\mu} \rightarrow x^{\mu}+\pi(x) \omega^{\mu} .
$$

As we shall see, this symmetry forbids the propagation of scalar fluctuations. Scalar theories invariant under this transformation inevitably lead to spontaneous breaking of Lorentz invariance, by turning on a nonvanishing scalar gradient $\partial_{\mu} \pi$. The vacuum of these theories selects a preferred direction that can be identified with the time coordinate, making this setup reminiscent of Einstein-Ether systems (see, e.g., [17] for a review). In our case, after Lorentz symmetry is spontaneously broken, no scalar excitations propagate. This result is not in contrast with the Lovelock theorem, where Lorentz invariance is assumed.

(ii) In Sec. III we couple scalar modes with vector fields in flat space, showing how to select interactions that-although we break the standard Abelian $U(1)$ symmetry-preserve a scalar-vector generalization of symmetry (1). The system spontaneously breaks Lorentz invariance, and the new symmetry can prevent the propagation of scalar excitations, leading to theories where only transverse vector modes are dynamical, even in the absence of Abelian $U(1)$ gauge invariance.

(iii) In Sec. IV we study four-dimensional scalar-tensor interactions in curved space, deriving them from a brane-world construction of a dynamical brane embedded in five-dimensional (5D) space [16]. We start in Sec. IV A turning gravity off, reviewing the arguments of $[14,16]$, and showing how symmetry (1) arises as a nonlinear realization of broken global symmetries in the 5D brane-world model. In Sec. IV B we then consider the case of dynamical gravity, showing that although symmetry (1) gets generally broken, nevertheless a novel continuous symmetry arises, inherited from 5D diffeomorphism invariance. In Sec. IV C we study consequences of this symmetry, providing sufficient conditions for avoiding the propagation of scalar modes around certain configurations. As a representative example, we demonstrate that no scalar excitations arise around a Schwarzschild solution, although the dynamics of spin-2 fluctuations is different with respect to Einstein gravity, making the theory distinguishable from GR. In Sec. IV D we study cosmology, pointing out that the structure of our theories leads to scalar constraints on cosmological configurations, leading to expanding spacetime geometries insensitive to the value of the cosmological constant. Correspondingly, we discuss some consequences for the dark energy problem. (iv) We conclude our discussion in Sec. V, followed by a technical Appendix.

\section{A SYMMETRY FOR SCALARLESS SCALAR THEORIES AROUND FLAT SPACE}

\section{A. The scalarless symmetry}

We start considering a class of scalar theories around flat space in the absence of gravity, whose Lagrangian densities are Lorentz invariant and lead to second order equations of motion for the scalar field $\pi(x)$. Besides a shift symmetry, we demand that these theories are invariant under the fielddependent coordinate transformation

$$
x^{\mu} \rightarrow x^{\mu}+\pi(x) \omega^{\mu}
$$

with $\omega^{\mu}$ a constant, infinitesimal 4-vector. This infinitesimal coordinate transformation acts on the scalar field $\pi(x)$ as

$\pi(x) \rightarrow \pi(x)+\delta_{\mathrm{sl}} \pi(x), \quad$ with $\quad \delta_{\mathrm{sl}} \pi(x) \equiv \frac{1}{2} \omega^{\mu} \partial_{\mu} \pi^{2}(x)$.

As we shall demonstrate soon, theories invariant under the symmetry (3) spontaneously break Lorentz symmetry, and the corresponding equations of motion require one to turn on a nonvanishing timelike background gradient $\partial_{\mu} \bar{\pi}$ whose norm satisfies the condition $\partial_{\mu} \bar{\pi} \partial^{\mu} \bar{\pi}<0$ (we work with "mostly plus" metric conventions).

On the other hand, the symmetry (3) prevents the propagation of linearized scalar excitations $\hat{\pi}$ around the background configuration $\bar{\pi}$. For this reason we dub transformation (3) scalarless (SL) symmetry. This fact was first noticed in [14], and we now reconsider it here. We select a background scalar field $\bar{\pi}(t)$ that spontaneously breaks Lorentz symmetry and solves the equations of motion. Splitting the scalar into a background homogenous configuration plus a small perturbation, $\pi(t, \vec{x})=\bar{\pi}(t)+\hat{\pi}(t, \vec{x})$, these two quantities transform under symmetry (3) as

$$
\begin{gathered}
\bar{\pi}(t) \rightarrow \bar{\pi}(t)+\omega^{0} \bar{\pi}(t) \dot{\bar{\pi}}(t), \\
\hat{\pi}(t, \vec{x}) \rightarrow \hat{\pi}(t, \vec{x})+\omega^{0} \partial_{0}[\bar{\pi}(t) \hat{\pi}(t, \vec{x})]+\bar{\pi}(t) \omega^{i} \partial_{i} \hat{\pi}(t, \vec{x}),
\end{gathered}
$$

for infinitesimal quantities $\omega^{\mu}$ and $\hat{\pi}$. This implies that, after fixing once for all the background configuration $\bar{\pi}(t)$, there remains a residual symmetry depending on the spatial components $\omega^{i}$ of the vector $\omega^{\mu}$ and acting on the small fluctuations as

$$
\hat{\pi}\left(x^{\mu}\right) \rightarrow \hat{\pi}\left(x^{\mu}\right)+\bar{\pi}(t) \omega^{i} \partial_{i} \hat{\pi}\left(x^{\mu}\right) .
$$

Such residual symmetry prevents a standard kinetic term for the fluctuations around Minkowski space: the contribution 
$(1 / 2)\left(\partial_{0} \hat{\pi}\right)^{2}$ to the scalar kinetic term containing time derivatives ${ }^{1}$ is not invariant under the residual symmetry (6); hence it cannot appear in the quadratic action for scalar fluctuations.

For these reasons, we regard scalar theories invariant under the SL symmetry (3) as a flat-space proxy for scalarless scalar-tensor systems in a unitary gauge, where the scalar profile is time dependent. For the rest of this section we characterize the structure and properties of these scalar theories to then explore their possible physical origin in the next sections.

\section{B. The scalarless scalar Lagrangians}

Are there shift-symmetric scalar theories invariant under the infinitesimal transformation (3)? The answer is affirmative, and these theories arise as a limit of a certain generalization of DBI-Galileons [16]: this fact was first shown in [14]. Here we streamline and elaborate on the analysis of [14].

To express more concisely the scalar systems we are interested in, we adopt the notation $\partial_{\mu} \pi=\pi_{, \mu}$ for denoting derivatives. In this section we raise and lower indexes with the Minkowski metric $\eta_{\mu \nu}$. We define

$$
X=-\pi_{, \mu} \pi^{, \mu}
$$

and the convenient combinations

$$
\begin{aligned}
& {\left[\Pi^{n}\right]=\pi^{, \mu}{ }_{, \alpha_{1}} \pi^{, \alpha_{1}}{ }_{, \alpha_{2}} \cdots \pi^{, \alpha_{n}}{ }_{, \mu},} \\
& {\left[\Phi^{n}\right]=\pi_{, \mu} \pi^{, \mu}{ }_{, \alpha_{1}} \cdots \pi^{, \alpha_{n}}{ }_{, \mu} \pi^{, \mu} .}
\end{aligned}
$$

The shift-symmetric Lagrangian densities describing the theories respecting the symmetry (3), up to total derivatives, are

$$
\begin{gathered}
\mathcal{L}_{1}=\sqrt{X}, \\
\mathcal{L}_{2}=\frac{1}{X}[\Phi], \\
\mathcal{L}_{3}=\frac{1}{\sqrt{X}}\left(\left[\Pi^{2}\right]-[\Pi]^{2}\right), \\
\mathcal{L}_{4}=\frac{1}{X}\left([\Pi]^{3}+2\left[\Pi^{3}\right]-3\left[\Pi^{2}\right][\Pi]\right) .
\end{gathered}
$$

\footnotetext{
${ }^{1}$ Instead, the contribution to the kinetic term containing spatial derivatives, $(\vec{\nabla} \pi)^{2}$, is invariant under the infinitesimal residual symmetry up to total derivatives, since

$$
\frac{1}{2} \partial_{j} \hat{\pi} \partial^{j} \hat{\pi} \rightarrow \partial_{j} \hat{\pi} \partial^{j}\left[\omega^{i} \partial_{i}(\bar{\pi}(t) \hat{\pi})\right]=\frac{\omega^{i}}{2} \partial_{i}\left[\bar{\pi}(t) \partial_{j} \hat{\pi} \partial^{j} \hat{\pi}\right] .
$$

We will explore in the next sections some consequences of this fact.
}

The Lagrangian (10) corresponds to a flat-space version of the cuscuton system [11], while Lagrangians (11)-(13) might be thought as higher derivative versions of this system (some common properties of all these Lagrangians will be discussed in what follows). These Lagrangians are weighted by appropriate powers of an energy scale (which we set to one) to provide the correct dimensionality.

We now present a more compact way to express and analyze these Lagrangians, which allows one to drive closer parallelisms with generalized Galileons, and more easily understand their symmetry properties. The previous four quantities - up to total derivatives and overall constantscan be expressed as

$$
\mathcal{L}_{n}=\sqrt{X} Y_{\mu}^{(\mathbf{n}) \mu}
$$

for $n=1, \ldots, 4$, where the tensors $Y_{\mu}^{(\mathbf{n}) \mu} \mathrm{read}$

$$
\begin{gathered}
Y_{\mu}^{(\mathbf{1}) \nu}=\epsilon_{\mu \alpha_{1} \beta_{1} \delta_{1}} \epsilon^{\nu \alpha_{1} \beta_{1} \delta_{1}}, \\
Y_{\mu}^{(\mathbf{2}) \nu}=\epsilon_{\mu \alpha_{1} \beta_{1} \delta_{1}} \epsilon^{\nu \alpha_{1} \beta_{1} \delta_{2}}\left(\pi^{, \delta_{1}} / \sqrt{X}\right)_{, \delta_{2}}, \\
Y_{\mu}^{(\mathbf{3}) \nu}=\epsilon_{\mu \alpha_{1} \beta_{1} \delta_{1}} \epsilon^{\nu \alpha_{1} \beta_{2} \delta_{2}}\left(\pi^{, \beta_{1}} / \sqrt{X}\right)_{, \beta_{2}}\left(\pi^{, \delta_{1}} / \sqrt{X}\right)_{, \delta_{2}}, \\
Y_{\mu}^{(\mathbf{4}) \nu}=\epsilon_{\mu \alpha_{1} \beta_{1} \delta_{1}} \epsilon^{\nu \alpha_{2} \beta_{2} \delta_{2}}\left(\pi^{, \alpha_{1}} / \sqrt{X}\right)_{, \alpha_{2}}\left(\pi^{, \beta_{1}} / \sqrt{X}\right)_{, \beta_{2}} \\
\times\left(\pi^{, \delta_{1}} / \sqrt{X}\right)_{, \delta_{2}} .
\end{gathered}
$$

The analogy with Galileon Lagrangians as expressed with the Levi-Civita symbols (see, e.g., [18,19] for early papers on these topics) is apparent. The traces of the tensors $Y_{\mu \nu}^{(\mathbf{n})}$ are total derivatives by themselves - they lead to nontrivial theories only when weighted by the overall $\sqrt{X}$ coefficient in Eq. (14).

Thanks to the antisymmetric properties of the LeviCivita symbols, the scalar equations of motion are at most second order, as it happens for generalized Galileons (see, e.g., $[20,21]$ for reviews). Once applying the SL infinitesimal transformation (3), one finds the relations

$$
\begin{aligned}
\delta_{\mathrm{sl}} \sqrt{X} & =\omega^{\rho} \partial_{\rho}(\pi \sqrt{X}), \\
\delta_{\mathrm{sl}}\left(\frac{\pi_{, \mu}}{\sqrt{X}}\right)_{, \nu} & =\omega^{\rho} \partial_{\rho}\left(\pi \frac{\pi_{, \mu}}{\sqrt{X}}\right)_{, \nu} .
\end{aligned}
$$

Making use of these results, of the chain rule, and the structure (14) of the Lagrangians, it is straightforward to realize that they transform under the infinitesimal SL transformation (3) as

$$
\delta_{\mathrm{sl}} \mathcal{L}_{n}=\omega^{\rho} \partial_{\rho}\left(\pi \mathcal{L}_{n}\right),
$$

for $n=1, \ldots, 4$. This is a total derivative, showing that the SL transformation (3) is a symmetry for these theories. 
After the work [14], these symmetries have been more recently reconsidered in related contexts in $[22,23]$ and recognized to be specific of the cuscuton Lagrangian $\sqrt{X}$ as Eq. (10). Here we emphasize that they are common to all the Lagrangians above (11)-(13).

\section{Spontaneous breaking of Lorentz symmetry and remarks on stability under radiative corrections}

Besides their symmetries, another important feature of Lagrangians (10)-(13) is their specific structure: they are nonpolynomial functions of $X$ (containing factors as $\sqrt{X}$ and $1 / X$ ), implying that the corresponding equations of motion do not allow for solutions with $X=0$. A consequence is that these systems spontaneously break Lorentz symmetry: although their Lagrangians are Lorentz invariant, the theories require vacua with a nonvanishing gradient for the scalar, with $\bar{\pi}_{, \mu} \neq 0$ (a well-defined square root $\sqrt{X}$ further requires $\bar{\pi}_{, \mu} \bar{\pi}^{\mu}<0$ ). Scalar backgrounds that depend only on time, $\bar{\pi}=\bar{\pi}(t)$, are special: making this choice of homogeneous background, the Lagrangians (12) and (13) vanish identically, while (10) and (11) become linear functions of the time derivatives $\dot{\bar{\pi}}$. Any linear combination of them with constant coefficients then reduces to a total derivative, ensuring that the homogeneous scalar configuration $\bar{\pi}(t)$ automatically satisfies the (trivial) equations of motion in Minkowski space and represents a consistent vacuum for the theory. For this reason, it is natural to concentrate on the choice $\bar{\pi}(t)$. This property of the Lagrangians of becoming linear in the scalar first derivative, once one focuses on homogeneous timedependent backgrounds, remains true also when the scalar system is coupled with other fields: we shall study some of its cosmological consequences in Sec. IV D.

Around homogeneous scalar backgrounds $\bar{\pi}(t)$ —as we proved in Sec. II A-no linearized scalar fluctuations propagate, making these scalar theories interesting. What should we expect about the stability of the structure of these theories under quantum effects? In the absence of dynamical fluctuations around homogeneous backgrounds, we might think that the structure of the Lagrangians is protected under quantum corrections associated with scalar modes-simply because no scalars propagate. When considering couplings with additional fields, one can induce symmetry breaking effects that render dynamical the scalar, making arguments of stability of the structure of the theory more subtle, also due to the absence of Lorentz invariance. Symmetry principles might help, as for the nonrenormalization theorems that characterize Galileons [24,25]. We defer this problem to a future work, although we make some additional remarks about this point in discussing cosmological models based on these theories in Sec. IV D.

In what comes next, we show that these scalar systems can arise in a variety of contexts, once continuous or global symmetries get broken in certain ways. Scalar modes-coupled with extra fields-can then arise as Goldstone bosons of broken symmetries. We start in Sec. III to discuss vector theories in Minkowski space with broken $U(1)$ symmetry, while in Sec. IV we consider scalar-tensor theories of gravity.

\section{SCALARLESS INTERACTIONS FROM BROKEN GAUGE SYMMETRIES}

In this section, we discuss how the scalarless SL symmetry of Sec. II A can characterize the physics of scalar excitations associated with broken gauge symmetries for vector degrees of freedom in flat space, again in the absence of gravity. This opens the possibility to study covariant theories that break explicitly gauge symmetries, without propagating scalar modes around appropriate backgrounds. For definiteness, we focus on the simplest case of a broken Abelian $U(1)$ gauge symmetry.

We consider the vector Lagrangian around flat space:

$$
\mathcal{L}_{V}=-\frac{1}{4} F_{\mu \nu} F^{\mu \nu}-V\left(A_{\mu}, \partial_{\rho} A_{\nu}\right),
$$

where $F_{\mu \nu}=\partial_{\mu} A_{\nu}-\partial_{\nu} A_{\mu}$ is the field strength of a vector field $A_{\mu}$, while $V\left(A_{\mu}, \partial_{\rho} A_{\nu}\right)$ is a vector potential. The vector potential generically breaks the Abelian gauge invariance

$$
A_{\mu} \rightarrow A_{\mu}+\partial_{\mu} \xi
$$

that is instead obeyed by the vector field strength $F_{\mu \nu}$ : this fact normally implies the propagation of a longitudinal scalar excitation, due to the gauge symmetry breaking potential $V$. In some cases, in an appropriate limit the Goldstone boson Lagrangian enjoys Galilean symmetries: this fact was first explored in [26-28]. Here we instead study possible realizations of the SL symmetry (3). For definiteness we focus on the vector Lagrangian (22) with a potential

$$
V=\beta m^{3} \sqrt{-\eta^{\mu \nu} A_{\mu} A_{\nu}},
$$

where $\beta$ is a dimensionless parameter and $m$ a mass scale providing the correct dimensionality. This potential explicitly breaks the $U(1)$ gauge symmetry (23); moreover, it is characterized by a square-root structure similar to the scalar Lagrangians investigated in Sec. II A.

In fact, our aim here is to exploit this similarity: given what we learned in the previous section, can we find new symmetries associated with the vector Lagrangian (22), which can prevent the propagation of linearized scalar modes? To answer this question more transparently, it is convenient to work with an equivalent system, making use of the Stuckelberg trick. We can reinstate the original gauge symmetry (23) introducing a scalar field $\pi$ in the combination 


$$
m A_{\mu} \rightarrow m A_{\mu}+\partial_{\mu} \pi
$$

and writing the scalar-vector potential

$$
V=\beta m^{2} \sqrt{-\eta^{\mu \nu}\left(m A_{\mu}+\partial_{\mu} \pi\right)\left(m A_{\nu}+\partial_{\nu} \pi\right)} .
$$

The system associated with the potential (26) is now invariant under the Abelian gauge symmetry

$$
\begin{gathered}
A_{\mu} \rightarrow A_{\mu}+\partial_{\mu} \xi, \\
\pi \rightarrow \pi-m \xi,
\end{gathered}
$$

for an arbitrary scalar function $\xi$. The scalar-vector potential (26) is distinct from the original one, Eq. (24). On the other hand, using the gauge symmetry (28) to fix the gauge $\pi=0$ one recovers the original Lagrangian (22) that is therefore physically equivalent to (26).

Here we make use of the symmetries Eqs. (27) and (28) and conveniently adopt the transverse Lorenz gauge $\partial^{\mu} A_{\mu}=0$ for describing the vector field dynamics. At this stage, the structure of the potential (26) would seem to imply that 3 degrees of freedom propagate in this system: 2 degrees of freedom in a gauge vector $A_{\mu}$ and 1 degree of freedom in the scalar $\pi$. On the other hand, considering Eq. (26) and taking the decoupling limit $m \rightarrow 0, \beta \rightarrow \infty$ such that $\beta m^{2}$ is kept finite, we get the scalar Lagrangian

$$
\mathcal{L}_{\mathrm{dec}}=\beta m^{2} \sqrt{-\eta^{\mu \nu} \partial_{\mu} \pi \partial_{\nu} \pi}
$$

which describes self-interactions of the Goldstone boson associated with the broken symmetry. Having taken $m \rightarrow 0$ this scalar Lagrangian loses the Abelian gauge symmetry (28); on the other hand, it enjoys the additional SL symmetry introduced in Sec. II, which prevents the propagation of scalar modes around time-dependent backgrounds $\bar{\pi}(t)$ that solve the equations of motion and spontaneously break Lorentz symmetry.

Such a decoupling limit argument suggests that the initial scalar-vector potential (26) is invariant under a more general scalar-vector global symmetry, even outside a decoupling limit. In fact, a straightforward computation along the arguments of Sec. II shows that the transformations (with $\omega^{\rho}$ an infinitesimal constant vector)

$$
\begin{gathered}
A_{\mu} \rightarrow A_{\mu}+\omega^{\rho} \partial_{\rho}\left(\pi A_{\mu}\right), \\
\pi \rightarrow \pi+\frac{1}{2} \omega^{\rho} \partial_{\rho} \pi^{2}
\end{gathered}
$$

leave the full potential (26) invariant up to total derivatives. This global symmetry is independent from $m$ and holds also outside the decoupling limit discussed above.
While we focused so far on the specific square root cuscuton potential (24), these very same considerations apply also to the vector versions of all the covariant scalar Lagrangians (11) - (13), which shall contain derivatives of the vector fields. It is sufficient to substitute the scalar first derivatives with the vector $A_{\mu}$ in those Lagrangians to determine covariant vector potentials. Since the resulting vector Lagrangians contain nonpolynomial functions as square roots and inverses of $A_{\mu} A^{\mu}$, consistent vacua around which fluctuations can be studied require a nonvanishing vacuum expectation value for $A_{\mu}$, or the more convenient Stuickelberg combination (25). The actual structure of the time-dependent vacuum expectation value will depend on the details of the complete theory, and possibly on the couplings of the vector with charged matter: a complete discussion goes beyond the scope of this section. (We will study in more detail this topic in the next section, when coupling scalars with gravity.) On the other hand, we notice that the simplest option is to choose the interactions so to select a time-dependent background for the Stückelberg field $\bar{\pi}(t)$. Linearized excitations around such a background are parametrized by hat quantities $A_{\mu}=\hat{A}_{\mu}$, $\pi(t \vec{x})=\bar{\pi}(t)+\hat{\pi}(t, \vec{x})$. The scalar fluctuations $\hat{\pi}$ enjoy the residual symmetry (6) which ensures that no scalar excitation propagates around this vacuum. ${ }^{2}$

In fact, the inevitable spontaneous breaking of Lorentz invariance of the initially covariant systems is the key to prevent the propagation of linearized scalar excitations around flat space, by exploiting the scalarless symmetry (3). Notice that these results are different from the partially massless vector theories of [29], where scalar excitations are forbidden by a new gauge symmetry in de Sitter space that arises when the vector mass is related with the de Sitter scale. On the other hand, it would be interesting to understand whether our arguments and techniques can be applied to theories of massive gravity, leading to examples of partially massless massive gravity theories in systems that spontaneously break Lorentz symmetry.

\section{SCALARLESS INTERACTIONS FROM BROKEN SPACETIME SYMMETRIES}

Besides broken gauge symmetries, another context where scalarless interactions can arise are theories breaking spacetime symmetries. In fact, previous investigations [14] based on [16] studied the possibility of obtaining these theories from broken global symmetries in fivedimensional brane worlds. In this section we analyze in

\footnotetext{
${ }^{2}$ Notice that the vector kinetic term $-F_{\mu \nu}^{2} / 4$ in Eq. (22) does not satisfy symmetry (30). On the other hand, this term preserves by itself the Abelian gauge symmetry which ensures that it does not excite scalar modes.

${ }^{3}$ See, e.g., [30] for an example of enhanced symmetry in a spontaneously broken Lorenz violating setting, for a Fierz-Pauli theory of massive gravity.
} 
more generality this topic and the consequences of coupling the scalar theories of Sec. II with gravity. Namely,

(i) In Sec. IVA we review the arguments of [14] and show that the scalarless SL symmetry (3) arises as a nonlinear realization of broken global symmetries in a five-dimensional brane-world context with a fixed spacetime geometry.

(ii) In Sec. IV B we turn on dynamical gravity and consistently couple our scalar Lagrangians (11)-(13) with tensor modes in curved space. This process generally breaks the SL symmetry that only holds in flat space; nevertheless we show that the scalar-tensor system obeys a novel continuous symmetry, inherited from broken diffeomorphism invariance in the fivedimensional brane-world model. Such new symmetry reduces to the SL symmetry in the flat-space limit.

(iii) In Sec. IV C we investigate the consequences of the new symmetry in the scalar-tensor system for the number of propagating degrees of freedom. In particular, we demonstrate that no linearized scalar modes propagate around spacetimes satisfying appropriate conditions, and then focus on the dynamics of fluctuations around spherically symmetric Schwarzschild black holes. We show that no additional degrees of freedom propagate besides the ones of GR. On the other hand, the dynamics of perturbations around black holes are different with respect to GR, making these theories distinguishable from Einstein gravity.

(iv) In Sec. IV D we focus on cosmology and discuss the possible relevance of our theories for the infrared dynamics of gravity and for the dark energy problem. We show that the effective action controlling homogeneous cosmological quantities acquires the form of a constrained system, and a constraint associated with the scalar field leads to expanding spacetime geometries which are insensitive to the value of the cosmological constant. Moreover, the system leads to self-accelerating configurations, with the rate of acceleration proportional to the parameters entering the scalar-tensor interactions.

\section{A. Embedding in flat five-dimensional brane-world models: The scalar as Goldstone boson of broken global symmetries}

A compelling physical motivation for the symmetry (3) and the Lagrangians of Eqs. (11)-(13) arises from a braneworld perspective. This brane-world point of view was first developed in [14], building on the results of [16] which introduced the DBI-Galileons. Here we briefly review this viewpoint $[14,16]$ and show that the scalar symmetry (3) arises as a nonlinear realization of a broken global spacetime symmetry in extradimensions.

We consider a four-dimensional brane whose worldvolume metric, $\gamma_{\mu \nu}$, is embedded in a five-dimensional bulk whose metric reads

$$
d s_{5 d}^{2}=\mathbf{g}_{M N} d X^{M} d X^{N}=\kappa_{0} \gamma_{\mu \nu} d x^{\mu} d x^{\nu}+d y^{2},
$$

where $y$ is the spatial extradimension. We introduce the constant parameter $\kappa_{0}$ as a constant "warp factor" that plays an important role for our arguments. We choose a foliation of the five-dimensional space expressing the brane embedding as

$$
\begin{gathered}
X^{\mu}=x^{\mu}, \\
X^{5}=\pi(x) .
\end{gathered}
$$

The scalar $\pi(x)$ geometrically parametrize fluctuations of the brane position in the fifth dimension. We then introduce four 5 -vectors as

$$
\begin{aligned}
e_{\mu}^{M} & =\frac{\partial X^{M}}{\partial x^{\mu}}, \\
& =\left(\ldots, \delta_{\mu}^{M}, \ldots, \partial_{\mu} \pi\right) .
\end{aligned}
$$

[For example, $e_{0}{ }^{M}=\left(1,0,0,0, \partial_{0} \pi\right)$.] These four 5vectors allow us to build the induced metric on the brane (or first fundamental form) as

$g_{\mu \nu}(x) \equiv e_{\mu}{ }^{M} e_{\nu}{ }^{N} \mathbf{g}_{M N}=\kappa_{0} \gamma_{\mu \nu}(x)+\partial_{\mu} \pi(x) \partial_{\nu} \pi(x)$.

Introducing a unit normal vector to the brane $n^{M}$, orthogonal to the vectors (35), one builds the second fundamental form - usually called extrinsic curvature — as the tensor

$$
\mathcal{K}_{\mu \nu}=e_{\mu}{ }^{M} e_{\nu}{ }^{N} \nabla_{M} n_{N}
$$

These are the tools needed to build the scalar theories we are investigating. Indeed, as proposed in [16], one can consider those Lagrangian densities on the brane world volume that lead to second order equations of motion for the fields involved. These are the Lovelock invariants [1] with the addition of Gibbons-Hawking-York terms on the brane [31,32], leading to the actions

$$
\begin{gathered}
\mathcal{S}_{1}=\frac{1}{\kappa_{0}^{3}} \int d^{4} x \sqrt{-g}, \\
\mathcal{S}_{2}=\frac{1}{\kappa_{0}^{2}} \int d^{4} x \sqrt{-g} \mathcal{K}, \\
\mathcal{S}_{3}=\frac{1}{\kappa_{0}} \int d^{4} x \sqrt{-g} R, \\
\mathcal{S}_{4}=\int d^{4} x \sqrt{-g} \mathcal{K}_{\mathrm{GB}},
\end{gathered}
$$

where $\mathcal{K}$ is the trace of the extrinsic curvature, $R$ is the Ricci scalar computed with the induced metric $g_{\mu \nu}$, while $\mathcal{K}_{\mathrm{GB}}$, the "boundary-term" for the five-dimensional 
Gauss-Bonnet combination [33], is given by $\mathcal{K}_{\mathrm{GB}}=$ $\mathcal{K K}_{\mu \nu}^{2}-2 \mathcal{K}_{\mu \nu}^{3} / 3-\mathcal{K}^{3} / 3-2 G^{\mu \nu} \mathcal{K}_{\mu \nu}$, with $G_{\mu \nu}$ the Einstein tensor evaluated with the metric $g_{\mu \nu}$. (The reason for the powers of $\kappa_{0}$ as overall coefficients will be clear soon.)

When expressing the actions of (38) in terms of the quantities $\gamma_{\mu \nu}$ and $\pi$ that appear in the expression for the induced metric (36), one obtains covariant scalar-tensor theories whose equations of motion are automatically at most second order. The scalar field $\pi$, in this perspective, plays the role of the Goldstone boson of broken global symmetries that are nonlinearly realized in the scalar theory under examination.

We now fix $\gamma_{\mu \nu}(x)=\eta_{\mu \nu}$ : the resulting scalar theories one obtains from Lagrangians (38) are the DBI-Galileons of [16]. While in this section we focus on broken global symmetries, in the next sections we shall examine how the scalars can nonlinearly realize broken continuous symmetries in curved spacetime. In this global case, the broken translation symmetry in the bulk is nonlinearly realized in terms of shift symmetry acting on the scalar field. Broken five-dimensional rotations are nonlinearly realized in terms of the infinitesimal symmetry transformation

$$
\pi \rightarrow \pi+\kappa_{0} \omega_{\mu} x^{\mu}+\pi \omega_{\mu} \partial^{\mu} \pi,
$$

for an arbitrary infinitesimal vector $\omega_{\mu}$. In the limit $\kappa_{0} \rightarrow 0$, the symmetry (42) boils down to the infinitesimal SL symmetry introduced in Eq. (3).

In fact, the limit $\kappa_{0} \rightarrow 0$ can be taken consistently [when including the appropriate powers of $\kappa_{0}$ as overall coefficients, as in Eq. (38)] and leads to the system (10)-(13). As shown in [14], these correspond to a particular case of DBI-Galileon actions, equipped with the symmetry (3) [or equivalently the $\kappa_{0} \rightarrow 0$ limit of (42)]. We refer the reader to [14] for more details on the $\kappa_{0} \rightarrow 0$ limit of the geometrical brane-world construction with a flat brane-world volume, and the associate global symmetries. In the next sections, we turn on gravity to study how the scalar $\pi$ can be related with broken continuous spacetime symmetries.

\section{B. Coupling the scalar theory with gravity: A new symmetry arises}

As first noticed in [16], a consistent coupling with gravity for the system discussed in Sec. IV A is straightforward: since actions (38) are built in terms of Lovelock invariants and Gibbons-Hawking-like terms, they are ensured to lead to second order equations of motion when expressed in terms of the constituents $\gamma_{\mu \nu}, \pi$ of the brane induced metric. In fact, taking the $\kappa_{0} \rightarrow 0$ limit of actions (38), one obtains special cases of Horndeski Lagrangian densities, which control possible interactions between the metric $\gamma_{\mu \nu}$ and the scalar $\pi$ :

$$
\mathcal{L}_{1}=\sqrt{X}
$$

$$
\begin{gathered}
\mathcal{L}_{2}=\frac{\pi_{; \mu} \Pi^{\mu \nu} \pi_{; \nu}}{X}, \\
\mathcal{L}_{3}=R+\frac{1}{X}\left(\left[\Pi^{2}\right]-[\Pi]^{2}\right), \\
\mathcal{L}_{4}=\frac{1}{X}\left([\Pi]^{3}+2\left[\Pi^{3}\right]-3\left[\Pi^{2}\right][\Pi]-12 \Pi_{\mu}{ }^{\rho} \pi_{; \rho} G^{\mu \nu} \pi_{; \nu}\right),
\end{gathered}
$$

where $\pi_{; \mu} \equiv \nabla_{\mu} \pi, \Pi_{\mu \nu} \equiv \nabla_{\mu} \nabla_{\nu} \pi, X \equiv-\pi^{; \mu} \pi_{; \mu}$, and indexes are raised/lowered with the dynamical metric $\gamma_{\mu \nu}$, which is also used to take covariant derivatives. The Ricci scalar $R$ and Einstein tensor $G_{\mu \nu}$ appearing in the previous expressions are computed in terms of the induced fourdimensional metric $\gamma_{\mu \nu}$.

As anticipated above, these theories lead to second order equations of motion, and belong to the class of Horndeski actions. Besides Horndeski systems, also special cases of beyond Horndeski or DHOST theories [34-37] can be obtained in terms of brane-induced scalar quantities, as pointed out in [15]. In particular, systems built in terms of the trace of powers of the extrinsic curvature $\operatorname{tr}\left[\mathcal{K}_{\mu \nu}^{n}\right], n \geq 2$ are known to lead to consistent covariant theories which avoid Ostrogradsky instabilities [36,38]. Before starting to analyze the properties of these systems, we can make some general comments:

(i) The Lagrangians (43)-(46) contain nonpolynomial functions of $X$ as $\sqrt{X}, 1 / X$; hence they spontaneously break Lorentz invariance, since consistent solutions of the equations of motion require a nonvanishing gradient for the scalar $\partial_{\mu} \bar{\pi} \neq 0$. In what follows we will be interested to explore the consequences of this fact.

(ii) The scalarless symmetry (3) is expected to break around curved spacetimes, since a covariantly constant vector $\omega^{\mu}$ generally does not exist outside flat space. Nevertheless, we shall learn that the scalartensor system obeys a novel continuous symmetry, inherited from broken diffeomorphism invariance in the five-dimensional brane-world model.

\section{A local symmetry inherited from higher dimensions}

The covariant scalar-tensor theories discussed above are expected to break symmetry (3) around generic curved configurations; on the other hand, we now discuss an additional symmetry for these systems, inherited from fivedimensional bulk continuous symmetries. This symmetry was already noticed in [15], without, however, realizing its higher dimensional origin.

We focus on the same five-dimensional brane-world perspective introduced in Sec. IVA. We consider the continuous symmetry associated with reparametrization invariance of the bulk spacetime, broken by the presence of the brane. The bulk coordinate transformations read as 


$$
X^{M} \rightarrow X^{M}-\chi^{M}\left(X^{N}\right),
$$

with $\chi^{M}$ denoting a five-dimensional vector with five independent components. The five-dimensional metric transforms as a tensor under this symmetry, which leads to the infinitesimal transformation

$$
\begin{aligned}
\mathbf{g}_{M N}(X) & \rightarrow \frac{\partial \tilde{X}^{P}}{\partial X^{M}} \frac{\partial \tilde{X}^{Q}}{\partial X^{N}} \mathbf{g}_{P Q}(\tilde{X}) \\
& =\mathbf{g}_{M N}+\chi^{L} \partial_{L} \mathbf{g}_{M N}+\partial_{M} \chi^{L} \mathbf{g}_{L N}+\partial_{N} \chi^{L} \mathbf{g}_{L M} .
\end{aligned}
$$

We get nonlinearly realized induced symmetries in the brane-world volume, controlled by the components of the 5 -vector $\chi^{A}$. We concentrate on vectors $\chi^{M}$ depending only on four-dimensional coordinates

$$
\chi^{M}=\left(\xi^{\mu}(x), \kappa_{0} \psi(x)\right),
$$

with $\psi(x)$ an arbitrary scalar field. This choice is made to preserve the standard four-dimensional diffeomorphism transformations. In fact, the first four components $\xi^{\mu}$ act on the induced metric $\gamma_{\mu \nu}$ and the scalar $\pi$ exactly as the standard coordinate invariance of general relativity, which we assume to hold in the covariant scalar-tensor system. The component $\chi^{5}=\kappa_{0} \psi$ provides us with a new symmetry transformation that acts on the metric and the scalar as

$$
\begin{aligned}
g_{\mu \nu}(x) & \rightarrow g_{\mu \nu}+\left(e_{\mu}{ }^{\rho} e_{\nu}^{5}+e_{\nu}{ }^{\rho} e_{\mu}^{5}\right) \partial_{\rho} \chi^{5} \mathbf{g}_{55} \\
& =\kappa_{0}\left(\gamma_{\mu \nu}+\partial_{\mu} \psi \partial_{\nu} \pi+\partial_{\mu} \pi \partial_{\nu} \psi\right)+\partial_{\mu} \pi \partial_{\nu} \pi,
\end{aligned}
$$

while

$$
\pi(x) \rightarrow \pi(x)-\kappa_{0} \psi(x) .
$$

In the limit $\kappa_{0} \rightarrow 0$ that, as we learned in Sec. IV A, is of most interest for us, the transformation acts on the tensor field $\gamma_{\mu \nu}$ only and reads

$$
\gamma_{\mu \nu} \rightarrow \gamma_{\mu \nu}+\partial_{\mu} \psi \partial_{\nu} \pi+\partial_{\mu} \pi \partial_{\nu} \psi,
$$

for an arbitrary, infinitesimal scalar function $\psi(x)$. The transformation (52) acting on the metric (and not on the scalar) is then a continuous infinitesimal symmetry for the covariant scalar-tensor actions (43)-(46).

As a concrete example, we consider the cuscuton system (43) minimally coupled with gravity, which we rewrite here as

$$
S_{1}=\int d^{4} x \sqrt{-\gamma} \sqrt{X}
$$

The argument of the integral transforms under the symmetry as

$$
\begin{aligned}
& \sqrt{-\operatorname{det} \gamma_{\mu \nu}} \sqrt{X} \\
& \rightarrow \sqrt{-\operatorname{det}\left(\gamma_{\mu \nu}+\partial_{\mu} \psi \partial_{\nu} \pi+\partial_{\mu} \pi \partial_{\nu} \psi\right)} \\
& \quad \times \sqrt{-\left(\gamma^{\mu \nu}-\partial^{\mu} \psi \partial^{\nu} \pi-\partial^{\mu} \pi \partial^{\nu} \psi\right) \partial_{\mu} \pi \partial_{\nu} \pi} \\
& =\sqrt{-\operatorname{det} \gamma_{\mu \nu}}(1+\partial \pi \cdot \partial \psi) \sqrt{X}(1-\partial \pi \cdot \partial \psi) \\
& =\sqrt{-\operatorname{det} \gamma_{\mu \nu}} \sqrt{X}
\end{aligned}
$$

where we kept terms up to linear order in the small scalar quantity $\psi$, showing as stated that action (43) is invariant under symmetry (52).

\section{Relating curved and flat space symmetries in the Minkowski limit}

We now argue that symmetry (52) might be regarded as a scalar-tensor, curved-space version of the infinitesimal scalar symmetry (3) introduced around flat space. To do so, we focus on fluctuations around a Minkowski geometry and assume a Lorentz-violating scalar background $\bar{\pi}$ whose gradient has a nonvanishing norm, say, $\partial_{\nu} \bar{\pi} \partial^{\nu} \bar{\pi}<0$; for definiteness, we choose $\bar{\pi}=t$. We aim to then relate (52) with (3). Symmetry (52) acting on infinitesimal metric fluctuations $h_{\mu \nu}$ around flat space (that is, $\gamma_{\mu \nu}=\eta_{\mu \nu}+h_{\mu \nu}$ ) reads

$$
h_{\mu \nu} \rightarrow h_{\mu \nu}+\partial_{\mu}\left(\psi \partial_{\nu} \bar{\pi}\right)+\partial_{\nu}\left(\psi \partial_{\mu} \bar{\pi}\right),
$$

which is equivalent to an infinitesimal coordinate transformation

$$
x^{\mu} \rightarrow x^{\mu}+\psi \partial^{\mu} \bar{\pi},
$$

for an arbitrary infinitesimal scalar quantity $\psi$. We stress, on the other hand, that the specific transformation (57) only acts on the tensor fluctuations $h_{\mu \nu}$, and not on the scalar field.

We can exploit the similarity of (56) with diffeomorphisms to "transfer" the symmetry transformation from the tensor to the scalar sector: we apply a standard infinitesimal diffeomorphism transformation to the metric fluctuation

$$
h_{\mu \nu} \rightarrow h_{\mu \nu}+\partial_{\mu} \xi_{\nu}+\partial_{\nu} \xi_{\mu}
$$

and choose $\xi_{\mu}=-\psi \partial^{\mu} \bar{\pi}$. The combination of the two transformations (56) and (58) compensates each other, leaving the metric fluctuations unchanged; on the other hand, the diffeomorphism transformation in (58) acts on the scalar fluctuations as well,

$$
\hat{\pi} \rightarrow \hat{\pi}+\xi^{\mu} \partial_{\mu} \bar{\pi}=\hat{\pi}-\psi\left(\partial^{\mu} \bar{\pi} \partial_{\mu} \bar{\pi}\right) .
$$


By choosing the free function $\psi$ as $\psi=\left(\bar{\pi} \omega^{i} \partial_{i} \hat{\pi}\right) / \bar{X}$, this transformation becomes identical to the flat-space residual symmetry (6) acting on scalar fluctuations. In this sense, the symmetry acting on the metric (52) is a scalar-tensor generalization of the flat-space scalar symmetry we started with in Sec. II A.

\section{Consequences of the symmetry for the propagating degrees of freedom}

We investigate the consequences of symmetry (52) for the number of propagating degrees of freedom around curved spacetimes, extending the arguments of [15].

Theories (43)-(46) lead to scalar background configurations that solve the equations of motion spontaneously breaking Lorentz symmetry, via a spacelike gradient for the background scalar $\bar{\pi}(x)$ such that $\partial_{\mu} \bar{\pi} \neq 0$. We study the dynamics of linearized fluctuations around background solutions. We split scalar and metric fluctuations as

$$
\begin{gathered}
\pi=\bar{\pi}+\hat{\pi}, \\
\gamma_{\mu \nu}=\bar{\gamma}_{\mu \nu}+\hat{h}_{\mu \nu},
\end{gathered}
$$

where the hat quantities are infinitesimal. Symmetry (52) does not act on the scalar (hence it leaves invariant the scalar background $\bar{\pi}$ ) and acts on metric fluctuations as

$$
\hat{h}_{\mu \nu} \rightarrow \hat{h}_{\mu \nu}+\bar{\nabla}_{\mu} \hat{\psi} \bar{\nabla}_{\nu} \bar{\pi}+\bar{\nabla}_{\nu} \hat{\psi} \bar{\nabla}_{\mu} \bar{\pi}
$$

with $\hat{\psi}(t \vec{x})$ an arbitrary infinitesimal scalar field. In certain circumstances, symmetry (62) ensures that scalar excitations do not propagate. To see this fact explicitly, we work with the action

$$
S=\int d^{4} x \sqrt{-\gamma}\left[M_{\mathrm{Pl}}^{2} R+\sum_{i} c_{i} \mathcal{L}_{i}\right],
$$

where $\mathcal{L}_{i}, i=1, \ldots, 4$ are the Lagrangian densities (43)-(46) and $c_{i}$ constant coefficients. We add for completeness the Einstein-Hilbert term $M_{\mathrm{Pl}}^{2} R$ that, of course, by itself does not introduce scalar excitations. We select background configurations that are solutions to the corresponding equations of motion with nonvanishing gradient $\bar{\nabla}_{\mu} \bar{\pi}$, and study the dynamics of small fluctuations defined as in Eqs. (60) and (61). The total action (63) is invariant under diffeomorphisms:

$$
\begin{gathered}
\hat{h}_{\mu \nu} \rightarrow \hat{h}_{\mu \nu}+\bar{\nabla}_{\mu} \hat{\xi}_{\nu}+\bar{\nabla}_{\nu} \hat{\xi}_{\mu}, \\
\hat{\pi} \rightarrow \hat{\pi}+\hat{\xi}^{\rho} \bar{\nabla}_{\rho} \bar{\pi},
\end{gathered}
$$

for an infinitesimal vector $\hat{\xi}^{\mu}$ (the covariant derivatives are taken with respect to the background metric $\bar{\gamma}_{\mu \nu}$ ). We can use this fact to "gauge away" the scalar fluctuations by selecting the gauge-fixing vector

$$
\hat{\xi}_{\mathrm{gf}}^{\mu}=-\hat{\pi}\left(\frac{\bar{\nabla}^{\mu} \bar{\pi}}{\bar{\nabla}^{\rho} \bar{\pi} \bar{\nabla}_{\rho} \bar{\pi}}\right) \equiv-\hat{\sigma} \bar{\nabla}^{\mu} \bar{\pi},
$$

where, for convenience, we collect pieces depending on the infinitesimal parts of the transformation into the scalar combination $\hat{\sigma}$. This choice of diffeomorphism vector $\hat{\xi}_{\mathrm{gf}}^{\mu}$ breaks diffeomorphism invariance fixing a gauge: while this is not an issue for the Einstein-Hilbert contribution to action (63) — which we know propagate only two tensor modes-it can be a problem for Lagrangians $\mathcal{L}_{i}$ : such a gauge choice "moves" scalar excitations from the scalar to the metric sector through the gauge transformation (64), which reads here

$$
\hat{h}_{\mu \nu} \rightarrow \hat{h}_{\mu \nu}-\bar{\nabla}_{\mu}\left(\hat{\sigma} \bar{\nabla}_{\nu} \bar{\pi}\right)-\bar{\nabla}_{\nu}\left(\hat{\sigma} \bar{\nabla}_{\mu} \bar{\pi}\right)
$$

On the other hand, recall that the scalar-tensor Lagrangians are invariant under symmetry (52): in cases where the scalar gradient $\bar{\nabla}_{\mu} \bar{\pi}$ is a Killing vector for the geometry

$$
\bar{\nabla}_{\mu} \bar{\nabla}_{\nu} \bar{\pi}=0,
$$

then-when choosing $\hat{\psi}=\hat{\sigma}$ - the symmetry transformation (62) exactly compensates diffeomorphism transformation (67), leaving the metric fluctuations unchanged. Hence, for background configurations satisfying the Killing condition (68) our symmetry arguments ensure that no scalar fluctuations propagate.

It is important to emphasize that our arguments do not imply that only configurations satisfying (68) avoid the propagation of scalar modes. In fact, there are studies based on Hamiltonian analysis that show that theories such as ours do not propagate scalar modes, once the scalar profile satisfies a unitary gauge, with no hypothesis on the metric profile $[12,13]$. In fact, our arguments provide sufficient conditions based on symmetries, not necessary ones: there can very well be situations where scalar modes do not propagate thanks to second-class constraints. Such second-class constraints might get "promoted" to first class-and then to local gauge symmetries-around certain configurations, as we are finding here.

We now proceed making a concrete example of spherically symmetric configurations where the symmetry prevents the propagation of scalar excitations, similarly to what happens in General Relativity (GR). On the other hand, differences with respect to GR arise in the propagation of tensor modes, whose properties are distinct from the ones of Einstein gravity.

\section{Spherically symmetric configurations}

The specific theories we examine admit various branches of spherically symmetric configurations, which have been 
studied in [15]. Solutions exist whose metric is identical to the ones of GR, and the scalar acquires a time-dependent profile, as in the configurations first discussed in [39,40]. Including for definiteness only Lagrangian $\mathcal{L}_{4}$ in (63), we consider the action

$S=M_{\mathrm{Pl}}^{2} \int d^{4} x \sqrt{-\gamma}\left[R+\lambda \sqrt{X}\left(R+\frac{1}{X}\left(\left[\Pi^{2}\right]-[\Pi]^{2}\right)\right)\right]$,

corresponding to a special case of quartic Horndeski, with $G_{4}=1+\lambda \sqrt{X}$; when choosing the dimensionless constant parameter $\lambda=0$, we recover the standard Einstein-Hilbert action. This theory admits a spherically symmetric solution whose metric corresponds to a Schwarzschild black hole; for our purposes it is convenient to express it in terms of Lemaitre coordinates, as proposed in the recent work [5]

$$
\begin{gathered}
\bar{\pi}=\bar{\Lambda} \tau, \\
d s^{2}=-d \tau^{2}+\frac{r_{s}}{r} d \rho^{2}+r^{2} d \Omega_{2}^{2},
\end{gathered}
$$

where $r_{s}=2 M, \bar{\Lambda}$ is an energy scale that from now on we set equal to one, and the quantity $r$ is expressed in terms of time and radial coordinates $(\tau, \rho)$ as

$$
r=r_{s}^{1 / 3}\left[\frac{3}{2}(\rho-\tau)\right]^{2 / 3} .
$$

The Horndeski function $G_{4}$ reads for this configuration $\bar{G}_{4}=1-\lambda \gamma^{00}\left(\partial_{0} \pi\right)^{2}=1+\lambda$. (Recall that we take $\bar{\Lambda}=1$.) The constant parameter $\lambda$ controls deviations from GR. A simple change of coordinates allows one to express the previous configuration in standard Schwarzschild coordinates; on the other hand, for our purposes it is convenient to make use of Lemaitre coordinates in which the scalar profile only depends (linearly) on time, and whose gradient satisfies the Killing condition (68).

Around the scalar-tensor configuration (70) and (71), then, no scalar excitations propagate for the arguments we developed above, but only the spin-2 tensor excitations of GR. On the other hand, the dynamics of linear fluctuations change with respect to GR, making this scalar-tensor theory distinguishable with respect to Einstein gravity. The study of scalar and metric fluctuations around black hole backgrounds with time-dependent scalar profiles was started in $[41,42]$, and stability issues in the scalar sector have been discussed in [3-7]. In our case, instabilities are absent because scalar modes do not propagate. We study the dynamics of tensor modes using the methods of [5] and relegate the technical analysis to Appendix.

As a representative example, we write here the linearized Lagrangian governing the single parity odd-excitation $\Psi$ for multipoles $\ell \geq 2$ (see Appendix for details):

$$
\begin{aligned}
\mathcal{L}_{\text {odd }}= & \frac{1}{2(1+\lambda)} \sqrt{\frac{r_{s}}{r}} \frac{r^{2}}{\ell(\ell+2)-2}\left[\dot{\Psi}^{2}-\frac{(1+\lambda) r}{r_{s}} \Psi^{\prime 2}\right. \\
& \left.+\left(\frac{15 r_{s}}{4 r^{3}}+\frac{(1+\lambda)}{4 r^{2}}(3-4 \ell(\ell+1))\right) \Psi^{2}\right],
\end{aligned}
$$

where the dot and prime indicate, respectively, derivatives along $\tau$ and $\rho$. When $\lambda=0$, this Lagrangian density leads to the Regge-Wheeler equation for parity-odd black hole fluctuations in Lemaitre coordinates. When $\lambda \neq 0$, instead, we notice that fluctuations propagate with a nonunit sound speed

$$
c_{\Psi}=1+\lambda,
$$

and the Regge-Wheeler potential is also affected in the contributions proportional to the multipole numbers $\ell$. For avoiding gradient instabilities, we must require $1+\lambda>0$.

This explicitly demonstrates that black hole fluctuations can allow one to distinguish GR from a gravity theory controlled by action (69), even if they admit spherically symmetric solutions identical to Einstein gravity and do propagate the same degrees of freedom. While we discussed here odd-parity modes, also even-parity mode dynamics is sensitive to the parameter $\lambda$, and in Appendix we explicitly demonstrate that scalar kinetic terms relative to scalar modes vanish. This does not indicate strong coupling problems - instead it is a consequence of the symmetry (62) discussed above.

\section{Constrained cosmology and the dark energy problem}

In this section we point out some additional features of the scalar-tensor systems under considerations, which make them interesting for characterizing the infrared properties of gravity. In the structure of the scalar-tensor interactions (43)-(46) the scalar $\pi$ formally appears with a single power in each of these Lagrangians (in the loose sense that a square root as $\sqrt{-(\partial \pi)^{2}}$ reduces the power from two to one). This structure is essential for developing the symmetry arguments as in the previous sections, but has the extra consequence that the scalar field, in certain circumstances, can act as the Lagrange multiplier for the scalartensor setup. Namely, its equation of motion can provide a constraint for the gravity sector of the theory, independent from additional couplings of gravity with other fields. This feature occurs when focusing in homogeneous timedependent configurations. In fact, we already remarked in Sec. II (in the absence of gravity) that when the scalar depends on time only, the Lagrangians become linear in $\dot{\pi}$, and this quantity becomes a constraint. When gravity is turned on, this linear dependence leads to a constraint condition for cosmological systems. We investigate this property in a specific example, aimed to explore its implications for the dark energy problem. 
We focus on the action

$$
\begin{aligned}
S= & \int d^{4} x \sqrt{-\gamma}\left\{M_{\mathrm{Pl}}^{2} R-6 \Lambda+\frac{\lambda}{2}\left[6 H_{0}^{2} \sqrt{X}+\sqrt{X} R\right.\right. \\
& \left.\left.+\frac{2}{X^{3 / 2}}\left(\partial_{\mu} \pi \Pi^{\mu \rho} \Pi_{\rho \sigma} \partial^{\sigma} \pi-\Pi \partial_{\mu} \pi \Pi^{\mu \rho} \partial_{\rho} \pi\right)\right]\right\} .
\end{aligned}
$$

The first line is the Einstein-Hilbert action equipped with a cosmological constant $\Lambda$; the second line contains the interactions we are interested in, weighted by an overall dimensionless constant $\lambda$. They include the cuscuton term $\sqrt{X}$, with an extra factor $H_{0}^{2}$ with a dimension of mass squared, and a specific nonminimal coupling with gravity in the degenerate higher order scalar theories (DHOST) class, selected so to ensure that tensors propagate with the speed of light around curved background configurations. (Notice that these specific interactions do not respect the symmetry discussed in Sec. IV B, but this is not relevant for the arguments of this section: here we only make use of the structure of the actions discussed in Sec. IV B.) These interactions have the property that the scalar appears linearly in the action. We specialize on a time-dependent, homogeneous background with a Friedmann-RobertsonWalker Ansatz for the metric

$$
\begin{gathered}
\pi=\bar{\pi}(t), \\
d s^{2}=-N^{2}(t) d t^{2}+a^{2}(t) d \vec{x}^{2} .
\end{gathered}
$$

We can derive the following effective action for the timedependent quantities $\pi(t), N(t)$, and $a(t)$ once the previous Ansatz is plugged into action (75):

$$
\begin{aligned}
S= & \mathcal{C} \int d t N(t) a^{3}(t)\left[\frac{H^{2}(t)}{N^{2}(t)}+\frac{\Lambda}{M_{\mathrm{Pl}}^{2}}\right. \\
& \left.-\frac{\lambda}{M_{\mathrm{Pl}}^{2}} \frac{\dot{\bar{\pi}}(t)}{2 N(t)}\left(H_{0}^{2}-\frac{H^{2}(t)}{N^{2}(t)}\right)\right] .
\end{aligned}
$$

The overall constant $\mathcal{C}$ includes the three-dimensional volume factor, and we denote $H=\dot{a} / a$. Both the lapse $N(t)$ and the scalar derivative $\dot{\pi}(t)$ act as Lagrange multipliers for the system. The two independent field equations for the scale factor and $\pi$ read

$$
\begin{gathered}
H^{2}(t)\left(1+\lambda \frac{\dot{\bar{\pi}}}{M_{\mathrm{Pl}}^{2}}\right)=\frac{\Lambda}{M_{\mathrm{Pl}}^{2}}, \\
H^{2}(t)+\frac{2}{3} \dot{H}(t)=H_{0}^{2} .
\end{gathered}
$$

The second equation is a constraint associated with the scalar Lagrange multiplier: it controls the Hubble parameter independently from the cosmological constant $\Lambda$. The solution for this system of equations is

$$
\begin{gathered}
H(t)=H_{0} \tanh \left[\frac{3 H_{0}}{2}\left(t+t_{0}\right)\right] \rightarrow H_{0}, \\
\dot{\bar{\pi}}(t)=\frac{-H_{0}^{2} M_{\mathrm{Pl}}^{2}+\Lambda \operatorname{coth}^{2}\left[\frac{3 H_{0}}{2}\left(t+t_{0}\right)\right]}{\lambda H_{0}^{2}} \rightarrow \frac{\Lambda-H_{0}^{2} M_{\mathrm{Pl}}^{2}}{\lambda H_{0}^{2}},
\end{gathered}
$$

with $t_{0}$ an integration constant, and the arrows indicate the late time asymptotics. At late times the Hubble parameter converges to the constant value $H_{0}$ independent from $\Lambda$, as expected from the constraint condition (80). Being a constrained field, the scalar profile accommodates as in expression (82) so to solve Eq. (79). Notice that in our discussion we did not include additional contributions in the form of matter or radiation; here we are only interested in cosmic acceleration, and we leave a study of realistic cosmology to a separate work. We nevertheless point out that the scalar can couple linearly to matter fields, preserving the property of the constraint equation above.

At what extent is this mechanism of use for addressing the dark energy problem? The ideas discussed above rely on two features, whose study goes beyond the scope of this work:

(1) As apparent from Eq. (81), the scale of late-time acceleration is controlled by the parameter $H_{0}$ entering as an overall factor in the cuscuton contribution to action (75). If it were for this term only, we would expect that the scalar is not dynamical thanks to the symmetries discussed in the previous sections. The scalar, on the other hand, couples to itself through DHOST contributions in (75), and possibly to matter fields in a realistic cosmological setting, making it dynamical in an expanding spacetime. Propagating fields - the scalar, graviton, or matter fields - can induce quantum loop corrections that renormalize the scale $H_{0}$ in the cuscuton action. It would be interesting to understand whether symmetries as the ones discussed in Sec. II can lead to nonrenormalization theorems similar to Galileons [24,25] which can protect the overall coefficients in the Lagrangian, even when they are softly broken in a cosmological setup [43].

(2) Besides a mechanism of self-acceleration, a distinctive feature of the mechanism above is that the bare cosmological constant $\Lambda$ does not contribute to the rate of expansion, thanks to the constraint condition (82). This result seems relevant for addressing the cosmological constant problem (see, e.g., $[8,44]$ for reviews). Whether it can lead to a realistic solution to the problem without fine-tuning is an interesting question: we notice here that this idea resembles the approach of unimodular gravity to solve the cosmological constant problem, where a constraint condition compensates a cosmological constant 
contribution to the field equations. Unimodular gravity does not solve the cosmological constant problem since operators that impose the constraint conditions (and control the rate of expansion) receive large quantum contributions equivalent to the cosmological constant problem, as shown in [45]. In our case, the analog question arises with respect to the stability of the parameter $H_{0}$ under loop corrections, as mentioned above.

We leave investigations on these points to future work.

\section{OUTLOOK}

In this work we developed the analysis of $[14,15]$, discussing how symmetry principles can be used for determining scalarless scalar interactions, which prevent the propagation of scalar fluctuations around certain backgrounds solving the equations of motion. We understood how scalarless interactions can arise when breaking gauge or spacetime symmetries in vector or tensor theories, with the scalar playing the role of Goldstone bosons of such symmetries. We have shown that such symmetries are generally associated with theories that spontaneously break Lorentz invariance by a nonvanishing scalar field gradient. In the scalar-tensor case, we provided sufficient conditions for ensuring that scalar perturbations do not propagate around appropriate background configurations equipped by Killing vectors. We then studied the consequences of our results for spherically symmetric configurations and for cosmology. We proved that scalar excitations do not propagate around black hole solutions; the dynamics of spin-2 modes, nevertheless, is different with respect to Einstein gravity, making these theories distinguishable from general relativity. In a cosmological setting, we noticed that these theories lead to constrained cosmological settings, with self-accelerating cosmological solutions in which the rate of cosmological expansion is independent from the value of a bare cosmological constant.

We leave many interesting questions to future work:

(i) Symmetry principles can limit the number of allowed interactions for the setup under examination and protect them under classical and quantum corrections. It would be interesting to understand how symmetries, even when broken in a spacetime setup as in Sec. IV D, can protect the size and structure of operators useful for addressing the dark energy problem.

(ii) Besides solutions with Killing vectors (see Sec. IV C), it would be interesting to find other contexts where the spacetime symmetry of Sec. IV B can be used to prevent the propagation of scalar fluctuations. A possibility is to use the high/low frequency splitting to study gravitational wave propagation in scalartensor theories as in [46] and to study whether symmetries acting on high-frequency modes forbid the propagation of scalar fluctuations. (iii) While in Sec. III we studied broken Abelian vector theories that do not propagate scalar modes, it would be interesting to investigate whether partially massless massive gravity theory exists, in systems that spontaneously break Lorentz symmetry.

\section{ACKNOWLEDGMENTS}

It is a pleasure to thank Ivonne Zavala for her careful reading of the manuscript. G. T. is partially funded by STFC Grant No. ST/P00055X/1.

\section{APPENDIX: SCALARLESS PERTURBATIONS AROUND BLACK-HOLE SPACETIMES}

In this Appendix we further develop the arguments of Sec. IV C. Using methods and results of [5], we study fluctuations around the spherically symmetric black hole configurations that are solutions to the field equations with spherically symmetric Ansatz associated with the action (we set here $M_{\mathrm{Pl}}=1$ )

$S=\int d^{4} x \sqrt{-\gamma}\left[R+\lambda \sqrt{X}\left(R+\frac{1}{X}\left(\left[\Pi^{2}\right]-[\Pi]^{2}\right)\right)\right]$,

which corresponds to quartic Horndeski with $G_{4}=1+\lambda \sqrt{X}$. A branch of spherically symmetric solutions is the Schwarzschild geometry written in Lemaitre coordinates as

$$
\begin{gathered}
\bar{\pi}=\tau, \\
d s^{2}=-d \tau^{2}+\frac{r_{s}}{r} d \rho^{2}+r^{2}\left(d \theta^{2}+\sin ^{2} \theta d \varphi^{2}\right),
\end{gathered}
$$

where $r_{s}$ is the Schwarzschild radius and

$$
r=r_{s}^{1 / 3}\left[\frac{3}{2}(\rho-\tau)\right]^{2 / 3} .
$$

Notice that the scalar profile depends on time only (and $\bar{X}=1$ ); hence its gradient is a Killing vector for the spacetime we consider. The symmetry arguments of the main text prevent the propagation of scalar degrees of freedom. We now show explicitly that the only dynamical degrees of freedom are the ones of GR; on the other hand, their evolution equations are distinct from the ones of Einstein gravity. As anticipated, we use the methods and results of the recent paper [5] which can be directly applied to our configuration, and we refer the reader to that work for the intermediate steps and for a clear discussion of the gauge conditions.

Linearized perturbations around spherically symmetric black holes are conveniently expanded in terms of spherical harmonics $Y_{\ell m}(\theta, \varphi)$. The modes separate into quantities that are odd or even under the parity symmetry $(\theta, \varphi) \rightarrow$ $(\pi-\theta, \pi+\varphi)$ (here $\pi$ denotes the number $3.1415 \ldots)$ that do not mix at the linearized level. We study them separately. 


\section{Odd parity fluctuations}

This class of fluctuations only includes metric perturbations, because scalar fluctuations are of even parity. There are then two modes to be analyzed (a third one can be gauged away using diffeomorphism invariance), usually denoted as $h_{(0)}^{\ell m}(\tau, \rho), h_{(1)}^{\ell m}(\tau, \rho)$. The metric can be decomposed at the linearized level as (we focus on odd parity fluctuations with $\ell \geq 2$ )

$$
\begin{aligned}
d s^{2}= & -d \tau^{2}+\frac{r_{s}}{r} d \rho^{2}+r^{2}\left(d \theta^{2}+\sin ^{2} \theta d \varphi^{2}\right) \\
& -2 d \tau d \theta \frac{1}{\sin \theta}\left[\sum_{\ell=2}^{\infty} \sum_{m=-\ell}^{\ell} h_{(0)}^{\ell m} \partial_{\varphi} Y_{\ell m}\right] \\
& +2 d \tau d \varphi \sin \theta\left[\sum_{\ell=2}^{\infty} \sum_{m=-\ell}^{\ell} h_{(0)}^{\ell m} \partial_{\theta} Y_{\ell m}\right] \\
& -2 d \rho d \theta \frac{1}{\sin \theta}\left[\sum_{\ell=2}^{\infty} \sum_{m=-\ell}^{\ell} h_{(1)}^{\ell m} \partial_{\varphi} Y_{\ell m}\right] \\
& +2 d \rho d \varphi \sin \theta\left[\sum_{\ell=2}^{\infty} \sum_{m=-\ell}^{\ell} h_{(1)}^{\ell m} \partial_{\theta} Y_{\ell m}\right]
\end{aligned}
$$

Plugging this decomposition into the action (A1), one gets the effective Lagrangian for the modes $h_{(0)}$ and $h_{(1)}$ [from now on we understand the multipole indexes $(\ell, m)$ in the $\left.h_{(i)}\right]$,

$$
\begin{aligned}
\mathcal{L}_{\text {odd }}= & \sqrt{\frac{r}{4 r_{s}}}\left(h_{(0)}^{\prime}-\dot{h}_{(1)}-\sqrt{\frac{4 r_{s}}{r^{3}}}\left(h_{(0)}+h_{(1)}\right)\right)^{2} \\
& +\frac{\ell(\ell+1)-2}{2}\left(\sqrt{\frac{r_{s}}{r}} h_{(0)}^{2}-(1+\lambda) \sqrt{\frac{r}{r_{s}}} h_{(1)}^{2}\right)^{2},
\end{aligned}
$$

$$
h_{\mu \nu}^{\text {even }}=\sum_{\ell} \sum_{m=-\ell}^{\ell}\left(\begin{array}{cc}
H_{(0)}^{\ell m} & H_{(1)}^{\ell m}(\tau, \rho) \\
H_{(1)}^{\ell m} & \frac{r_{s}}{r} H_{(2)}^{\ell m}(\tau, \rho) \\
\alpha^{\ell m} \nabla_{A} & \beta^{\ell m} \nabla_{A}
\end{array}\right.
$$

respectively, in the scalar and metric sector, and $g_{A B}$ parametrize the metric on the two-sphere (the capital Latin indexes denote coordinates on such a sphere). Understanding again the indexes $(\ell, m)$, the quantities $\hat{\pi}$ (scalar fluctuation), and $H_{(0)}, H_{(1)}, H_{(2)}, K, Q, \alpha, \beta$ (parity even metric fluctuations) depend on $\tau$ and $\rho$. Some of these quantities can be set to zero exploiting diffeomorphism invariance.

We shall now separate the discussion for modes with $\ell=0,1$ and $\ell \geq 2$ with the specific aim to demonstrate the with dot and prime denoting derivatives along $\tau$ and $\rho$, respectively. Notice that the scalar-tensor interactions associated with the additional coupling $\lambda$ in action (A1) only controls the coefficient depending on $\lambda$ in the last term of Eq. (A6). The two quantities $h_{(0)}$ and $h_{(1)}$ are not independent: the corresponding field equations can be combined so to obtain a constraint between the two fields that can be solved by reexpressing the two quantities in terms of a single field $\Psi$ as follows:

$$
\begin{array}{r}
h_{(0)}=\frac{1}{\ell(\ell+1)-2} \frac{r^{2}}{r_{s}}\left(r \Psi^{\prime}+\frac{5}{2} \sqrt{\left.\frac{r_{s}}{r} \Psi\right),}\right. \\
h_{(1)}=\frac{1}{\ell(\ell+1)-2} \frac{r}{1+\lambda}\left(r \dot{\Psi}-\frac{5}{2} \sqrt{\left.\frac{r_{s}}{r} \Psi\right) .}\right.
\end{array}
$$

Plugging these expressions in Eq. (A6) one finds

$$
\begin{aligned}
\mathcal{L}_{\text {odd }}= & \frac{1}{2(1+\lambda)} \sqrt{\frac{r_{s}}{r}} \frac{r^{2}}{\ell(\ell+2)-2}\left[\dot{\Psi}^{2}-\frac{(1+\lambda) r}{r_{s}} \Psi^{\prime 2}\right. \\
& \left.+\left(\frac{15 r_{s}}{4 r^{3}}+\frac{(1+\lambda)}{4 r^{2}}(3-4 \ell(\ell+1))\right) \Psi^{2}\right],
\end{aligned}
$$

that we discussed as Eq. (73) in the main text.

\section{Even parity fluctuations}

Scalar excitations can contribute to even parity perturbations that we parametrize as

$$
\pi(x)=\tau+\sum_{\ell} \sum_{m=-\ell}^{\ell} \hat{\pi}^{\ell m}(\tau, \rho) Y_{\ell m}(\theta, \varphi),
$$

absence of additional scalar modes with respect to GR, thanks to the symmetry as discussed in the main text in Sec. IV.

\section{a. Monopole fluctuations, $\ell=0$}

For the monopole sector, the fluctuations $\alpha, \beta, Q$ are absent, while we can set to zero $H_{0}$ and $K$ with a diffeomorphism transformation. The resulting effective Lagrangian reads (recall that we use conventions where 
the dot and prime denote, respectively, derivatives along $\tau$ and $\rho$ )

$$
\begin{aligned}
\mathcal{L}_{\text {even }}^{\ell=0}= & 2 \lambda \sqrt{\frac{r_{s}}{r}} \hat{\pi}^{\prime 2}+\frac{1+\lambda}{2} \sqrt{\frac{r_{s}}{r}} H_{2}^{2} \\
& -2 \lambda \sqrt{\frac{r_{s}}{r}} H_{2} \hat{\pi}^{\prime}+2 r H_{1} \dot{H}_{2} .
\end{aligned}
$$

$H_{1}$ is a Lagrange multiplier that imposes the condition $\mathrm{H}_{2}=0$ (up to a contribution independent from time that can be readsorbed in the background). Using this condition, we get

$$
\mathcal{L}_{\text {even }}^{\ell=0}=2 \lambda \sqrt{\frac{r_{s}}{r}} \pi^{\prime 2}
$$

Hence the monopole scalar fluctuation does not propagate, since $\hat{\pi}$ only appears in the effective with spatial derivatives. This is a consequence of the symmetry arguments discussed in the main text.

\section{b. Dipole fluctuations, $\ell=1$}

In this case, a convenient gauge to choose is

$$
H_{0}=\beta=r^{2} K-Q=0 .
$$

The effective Lagrangian for $\ell=1$ fluctuations is

$$
\begin{aligned}
\mathcal{L}_{\text {even }}^{\ell=1}= & 2 \lambda \sqrt{\frac{r_{s}^{3}}{r^{3}}}\left(\frac{r}{r_{s}} \hat{\pi}^{\prime 2}-\frac{\hat{\pi}^{2}}{r^{2}}\right)+\frac{1+\lambda}{2} \sqrt{\frac{r_{s}}{r}} H_{2}^{2} \\
& +2 \sqrt{\frac{r_{s}}{r}} H_{2}\left[(r \alpha)-\lambda r\left(\frac{\hat{\pi}}{r}\right)^{\prime}\right]+\sqrt{\frac{r_{s}}{r}} H_{1}^{2} \\
& +2 H_{1}\left(r \dot{H}_{2}-\sqrt{\frac{r_{s}}{r}} \alpha^{\prime}\right)+\sqrt{\frac{r_{s}}{r}} \alpha^{\prime 2} .
\end{aligned}
$$

The field $H_{1}$ is nondynamical, and it is fixed by its equation of motion to

$$
H_{1}=-\sqrt{r_{s} r} \dot{H}_{2}+\alpha^{\prime}
$$

Plugging this condition in the Lagrangian, one finds that the field $\alpha$ becomes a Lagrange multiplier, imposing the condition

$$
r \dot{H}_{2}^{\prime}+2 \sqrt{\frac{r_{s}}{r}} \dot{H}_{2}+\frac{3 r_{s}}{2 r^{2}} H_{2}=0 .
$$

Using this information, the effective Lagrangian results:

$$
\begin{aligned}
\mathcal{L}_{\text {even }}^{\ell=1}= & 2 \lambda \sqrt{\frac{r_{s}^{3}}{r^{3}}}\left(\frac{r}{r_{s}} \hat{\pi}^{\prime 2}-\frac{\hat{\pi}^{2}}{r^{2}}\right)+\lambda \sqrt{\frac{r_{s}}{r}} \hat{\pi}\left(2 H_{2}^{\prime}+\sqrt{\frac{r_{s}}{r}} H_{2}\right) \\
& -\sqrt{r_{s} r^{3}} \dot{H}_{2}^{2}+\sqrt{\frac{r_{s}}{4 r}} H_{2}^{2},
\end{aligned}
$$

where the field $\mathrm{H}_{2}$ satisfies the constraint condition (A16). The scalar field $\hat{\pi}$ is nondynamical since it appears with no time derivatives in the effective Lagrangian; hence also monopole fluctuations do not rise to dynamical degrees of freedom.

\section{c. Multipole fluctuations with $\ell \geq \mathbf{2}$}

For higher multipoles the analysis is more involved, since in this case there are propagating tensor degrees of freedom (as in Einstein gravity) whose dynamics is influenced by the nonpropagating scalar modes. It is hard to fully diagonalize the equations of motion; on the other hand, we shall prove that there are no additional degrees of freedom with respect to GR.

A convenient gauge is

$$
H_{0}=K=Q=0 .
$$

We are left with five fields to analyze: $H_{1}, H_{2}, \alpha, \beta, \pi$. Exactly as for the monopole case $\ell=1$, the field $H_{1}$ is nondynamical and is algebraically determined by its equations of motion. Plugging this information in the effective Lagrangian, one finds that also in this case $\alpha$ leads to a Lagrange multiplier that imposes the condition

$$
\begin{gathered}
r \dot{H}_{2}^{\prime}+\left(\frac{\ell(\ell+1)}{2}+1\right) \sqrt{\frac{r_{s}}{r}} \dot{H}_{2}+\frac{3 \ell(\ell+1) r_{s}}{4 r^{2}} H_{2} \\
=\frac{\ell(\ell+1)}{2}\left[2 \sqrt{\frac{r}{r_{s}}} \dot{\beta}^{\prime}+\frac{3}{r} \dot{\beta}+\frac{\beta^{\prime}}{r}+\frac{3}{r^{2}} \sqrt{\frac{r_{s}}{r}} \beta\right] .
\end{gathered}
$$

This Lagrange constraint (A19) is not easy to immediately interpret, but it says that the quantity $\mathrm{H}_{2}$ is not an independent field. This can be made more manifest by the change of variable from $\beta$ to $\gamma$,

$$
\beta \equiv \gamma+\frac{\sqrt{r_{s} r}}{\ell(\ell+1)} H_{2}
$$

which allows us to reexpress the constraint (A19) as

$\partial_{\tau}\left[\frac{H_{2}}{r^{3 / 2}}\right]=\frac{3 \ell(\ell+1)}{\sqrt{r_{s}}\left(\ell^{2}+\ell-2\right)}\left[\frac{2}{3 \sqrt{r_{s}}} \partial_{\tau}\left(\frac{\gamma^{\prime}}{\sqrt{r}}\right)+\frac{1}{r} \partial_{\tau}\left(\frac{\gamma}{r}\right)\right]$.

Although we do not use the field $\gamma$ in what comes next, relation (A21) demonstrates that the field $\mathrm{H}_{2}$ can be expressed in terms of this quantity. (Notice that since $r$ 
depends on $\tau$ [see Eq. (A4)], it is nevertheless not immediate to integrate (A21).)

Using the previous information, the effective Lagrangian for the modes $\pi, H_{2}, \beta$ results in

$$
\begin{aligned}
\mathcal{L}_{\mathrm{even}}^{\ell \geq 2}= & \frac{1-\ell-\ell^{2}}{2} \sqrt{\frac{r}{r_{s}}} \dot{\beta}^{2}+2 r \dot{H}_{2} \dot{\beta}-\frac{2}{\ell(\ell+1)} \sqrt{\frac{r^{4}}{r_{s}}} \dot{H}_{2}^{2} \\
& -2 \sqrt{\frac{r}{r_{s}}} \beta \dot{H}_{2}+2 \lambda \sqrt{\frac{r}{r_{s}}} \pi^{\prime 2}+\lambda \frac{2 \ell(\ell+1)}{r} \beta \hat{\pi}^{\prime} \\
& -2 \lambda \sqrt{\frac{r}{r_{s}}} H_{2} \pi^{\prime}-\frac{\ell(\ell+1)}{r^{2}}\left(\sqrt{\frac{r}{r_{s}}} \beta^{2}+r \beta H_{2}\right. \\
& \left.+2 \lambda \sqrt{\frac{r}{r_{s}}} \beta \hat{\pi}-2 \lambda r_{s} H_{2} \hat{\pi}+\lambda \sqrt{\frac{r^{3}}{r_{s}^{3}}} \hat{\pi}^{2}\right) \\
& +\frac{1+\lambda}{2} \sqrt{\frac{r}{r_{s}}} H_{2}^{2},
\end{aligned}
$$

where the quantities $\mathrm{H}_{2}$ and $\beta$ are related by the Lagrange constraint (A19), or alternatively the relation (A21), which shows that $\mathrm{H}_{2}$ is not an independent field. Then Lagrangian (A22) contains two independent modes, $\beta$ and $\pi$. On the other hand, the scalar $\hat{\pi}$ does not appear with time derivatives, and it is not difficult to realize that its equation of motion provides a constraint, algebraically fixing $\pi$ in terms of the remaining quantities. This fact is more easily seen shifting $\mathrm{H}_{2}$ to $\mathrm{K}_{2}$ by the following relation:

$$
H_{2} \equiv K_{2}-2 \ell(\ell+1) \sqrt{\frac{r_{s}}{r^{3}}} \pi+2 \pi^{\prime}
$$

When $\lambda \neq 0$, the equation of motion for $\pi$ provides the following constraint, when expressed in terms of $H_{2}, \beta$ :

$$
\begin{aligned}
& \ell(\ell+1)\left(2 \ell^{2}+2 \ell-r\right) \pi \\
& \quad=\sqrt{\frac{r^{3}}{4 r_{s}}}(2 \ell(\ell+1)-1) K_{2}+\frac{r^{3}}{r_{s}} K_{2}^{\prime}+\sqrt{\frac{r^{5}}{r_{s}^{3}}} \ell(\ell+1) \beta^{\prime} .
\end{aligned}
$$

This relation-as well as (A21) - demonstrates that the effective Lagrangian (A22) describes the dynamics of a single propagating degree of freedom, as in Einstein gravity, and our scalar-tensor interactions do not excite additional modes around our black hole configuration, as expected by our symmetry arguments.
[1] D. Lovelock, The Einstein tensor and its generalizations, J. Math. Phys. (N.Y.) 12, 498 (1971).

[2] P. Creminelli, M. Lewandowski, G. Tambalo, and F. Vernizzi, Gravitational wave decay into dark energy, J. Cosmol. Astropart. Phys. 12 (2018) 025.

[3] H. Ogawa, T. Kobayashi, and T. Suyama, Instability of hairy black holes in shift-symmetric Horndeski theories, Phys. Rev. D 93, 064078 (2016).

[4] K. Takahashi and T. Suyama, Linear perturbation analysis of hairy black holes in shift-symmetric Horndeski theories: Odd-parity perturbations, Phys. Rev. D 95, 024034 (2017).

[5] J. Khoury, M. Trodden, and S. S. C. Wong, Existence and instability of novel hairy black holes in shift-symmetric Horndeski theories, arXiv:2007.01320.

[6] E. Babichev, C. Charmousis, G. Esposito-Farse, and A. Lehbel, Stability of Black Holes and the Speed of Gravitational Waves Within Self-Tuning Cosmological Models, Phys. Rev. Lett. 120, 241101 (2018).

[7] C. de Rham and J. Zhang, Perturbations of stealth black holes in degenerate higher-order scalar-tensor theories, Phys. Rev. D 100, 124023 (2019).

[8] S. Weinberg, The cosmological constant problem, Rev. Mod. Phys. 61, 1 (1989).

[9] C. Lin and S. Mukohyama, A class of minimally modified gravity theories, J. Cosmol. Astropart. Phys. 10 (2017) 033.
[10] K. Aoki, C. Lin, and S. Mukohyama, Novel matter coupling in general relativity via canonical transformation, Phys. Rev. D 98, 044022 (2018).

[11] N. Afshordi, D. J. H. Chung, and G. Geshnizjani, Causal field theory with an infinite speed of sound, Phys. Rev. D 75, 083513 (2007).

[12] A. Iyonaga, K. Takahashi, and T. Kobayashi, Extended cuscuton: Formulation, J. Cosmol. Astropart. Phys. 12 (2018) 002.

[13] X. Gao and Z.-B. Yao, Spatially covariant gravity theories with two tensorial degrees of freedom: The formalism, Phys. Rev. D 101, 064018 (2020).

[14] J. Chagoya and G. Tasinato, A geometrical approach to degenerate scalar-tensor theories, J. High Energy Phys. 02 (2017) 113.

[15] J. Chagoya and G. Tasinato, A new scalartensor realization of Horava-Lifshitz gravity, Classical Quantum Gravity 36, 075014 (2019).

[16] C. de Rham and A. J. Tolley, DBI and the Galileon reunited, J. Cosmol. Astropart. Phys. 05 (2010) 015.

[17] T. Jacobson, Einstein-aether gravity: A status report, Proc. Sci., QG-PH2007 (2007) 020 [arXiv:0801.1547].

[18] D. B. Fairlie, J. Govaerts, and A. Morozov, Universal field equations with covariant solutions, Nucl. Phys. B373, 214 (1992).

[19] D. B. Fairlie and J. Govaerts, Euler hierarchies and universal equations, J. Math. Phys. (N.Y.) 33, 3543 (1992). 
[20] C. Deffayet and D. A. Steer, A formal introduction to Horndeski and Galileon theories and their generalizations, Classical Quantum Gravity 30, 214006 (2013).

[21] C. de Rham, Galileons in the Sky, C.R. Phys. 13, 666 (2012).

[22] E. Pajer and D. Stefanyszyn, Symmetric superfluids, J. High Energy Phys. 06 (2019) 008.

[23] T. Grall, S. Jazayeri, and E. Pajer, Symmetric scalars, J. Cosmol. Astropart. Phys. 05 (2020) 031.

[24] M. A. Luty, M. Porrati, and R. Rattazzi, Strong interactions and stability in the DGP model, J. High Energy Phys. 09 (2003) 029.

[25] A. Nicolis and R. Rattazzi, Classical and quantum consistency of the DGP model, J. High Energy Phys. 06 (2004) 059.

[26] G. Tasinato, Cosmic acceleration from Abelian symmetry breaking, J. High Energy Phys. 04 (2014) 067.

[27] L. Heisenberg, Generalization of the proca action, J. Cosmol. Astropart. Phys. 05 (2014) 015.

[28] G. Tasinato, A small cosmological constant from Abelian symmetry breaking, Classical Quantum Gravity 31, 225004 (2014).

[29] S. Deser and R. I. Nepomechie, Gauge invariance versus masslessness in De Sitter space, Ann. Phys. (N.Y.) 154, 396 (1984).

[30] G. Tasinato, K. Koyama, and G. Niz, New symmetries in Fierz-Pauli massive gravity, J. High Energy Phys. 07 (2012) 062.

[31] J. W. York, Jr., Role of Conformal Three Geometry in the Dynamics of Gravitation, Phys. Rev. Lett. 28, 1082 (1972).

[32] G. W. Gibbons and S. W. Hawking, Action integrals and partition functions in quantum gravity, Phys. Rev. D 15, 2752 (1977).

[33] S. C. Davis, Generalized Israel junction conditions for a Gauss-Bonnet brane world, Phys. Rev. D 67, 024030 (2003).

[34] D. Langlois and K. Noui, Degenerate higher derivative theories beyond Horndeski: Evading the Ostrogradski instability, J. Cosmol. Astropart. Phys. 02 (2016) 034.
[35] D. Langlois and K. Noui, Hamiltonian analysis of higher derivative scalar-tensor theories, J. Cosmol. Astropart. Phys. 07 (2016) 016.

[36] M. Crisostomi, K. Koyama, and G. Tasinato, Extended scalar-tensor theories of gravity, J. Cosmol. Astropart. Phys. 04 (2016) 044.

[37] C. de Rham and A. Matas, Ostrogradsky in theories with multiple fields, J. Cosmol. Astropart. Phys. 06 (2016) 041.

[38] M. Crisostomi, M. Hull, K. Koyama, and G. Tasinato, Horndeski: Beyond, or not beyond?, J. Cosmol. Astropart. Phys. 03 (2016) 038.

[39] E. Babichev and C. Charmousis, Dressing a black hole with a time-dependent Galileon, J. High Energy Phys. 08 (2014) 106.

[40] T. Kobayashi and N. Tanahashi, Exact black hole solutions in shift symmetric scalar-tensor theories, Prog. Theor. Exp. Phys. 2014, 73E02 (2014).

[41] T. Kobayashi, H. Motohashi, and T. Suyama, Black hole perturbation in the most general scalar-tensor theory with second-order field equations: The odd-parity sector, Phys. Rev. D 85, 084025 (2012).

[42] T. Kobayashi, H. Motohashi, and T. Suyama, Black hole perturbation in the most general scalar-tensor theory with second-order field equations. II. The even-parity sector, Phys. Rev. D 89, 084042 (2014).

[43] D. Pirtskhalava, L. Santoni, E. Trincherini, and F. Vernizzi, Weakly broken Galileon symmetry, J. Cosmol. Astropart. Phys. 09 (2015) 007.

[44] A. Padilla, Lectures on the cosmological constant problem, arXiv:1502.05296.

[45] A. Padilla and I. D. Saltas, A note on classical and quantum unimodular gravity, Eur. Phys. J. C 75, 561 (2015).

[46] A. Garoffolo, G. Tasinato, C. Carbone, D. Bertacca, and S. Matarrese, Gravitational waves and geometrical optics in scalar-tensor theories, arXiv:1912.08093. 